\title{
Buprestides recueillis par M. E. von Bodemeyer en Perse et en Asie mineure.
}

Par Ch. Kerremans, Bruxelles.

M. E. von Bodemeyer a bien voulu me communiquer une partie des Buprestides recueillis au cours de ses longues et remarquables recherches entomologiques en Perse et en Asie mineure. Le lot considérable de Sphenoptera qu'il a soumis à mon examen constitue un ensemble unique tant par le nombre des espèces que par celui des exemplaires, recueillis pour la plupart dans le Luristan, province occidentale de la Perse et touchant aux frontières de l'Asie mineure. Les espèces de ce genre sont rarement en nombre parmi les récoltes entomologiques, et cela tient surtout à leur mode de vie, presque souterrain, que M. von Bodemeyer a eu l'occasion de constater, durant une période de près de douze ans. Sur les plateaux les plus dénudés et où il n'espérait rien recueillir, il lui suffisait de faire déraciner quelques Onobrychis pour trouver autour de leurs racines quantité de Sphenoptera. Plus de quarante espèces ont été recueillies ainsi et, pour la plupart, représentées par de longues séries d'individus, offrant de grandes variations quant à la taille et à la sculpture générale.

Pour le moment, je me contente d'en dresser la liste et de décrire celles que je crois nouvelles, laissant à chacune des espèces le nom qui lui revient; mais je crains fort que, dans la suite, certains de ces noms tomberont en synonymie.

Bien des espèces du genre Sphenoptera ont été décrites sur le vu de spécimens uniques et fondées sur des caractères sujets à varier. Ce n'est que l'examen des types épars dans les collections qui permettra de fixer les opinions. Je réserve donc pour ma Monographie la critique des espèces.

Mais il y a lieu de féliciter le vaillant entomologiste voyageur pour avoir aussi largement augmenté nos connaissances sur la faune de l'Orient et, personnellement, je le remercie de m'avoir permis d'étendre mes recherches sur le même sujet.

\section{Pars I.}

1. Julodis Iris Cast., Mongr., t. 1 (1835), p. 12, pl. 3, f. 14. L'espèce s'étend jusqu'en Arabie, en Egypte et en Asie mineure à l'ouest; vers l'est, elle atteint la Kirghisie, le Turkestan et l'Inde. Les exemplaires recueillis par M. von Bodemeyer proviennent de la province du Luristan (Perse). 
2. Julodis intricata Redt., Russegg. Reis., t. 2 (1843), p. 982, pl. A, f. 10. - Espèce très caractéristique par son faciès trapu à dessus très convexe. - Perse: Luristan. Habite aussi l'Asie mineure (Anatolie).

3. Julodis levicostata Gory, Monogr. Supp., t. 4 (1840), p. 16, pl. 3, f. 14, ainsi que la variété ramifera Mars., L'Abeille, t. 2 (1865), p. 71; pris tous deux en assez grande quantité dans le Luristan (Perse).

4. Julodis ampliata Mars., L'Abeille, t. 2 (1865), p. 66 . Constitue la forme extrême de l'onopordi Fab. Très variable au point de vue de la taille et de la coloration. Très nombreux exemplaires recueillis en Luristan (Perse), depuis le luteogramma Mars., à faciès moins robuste, jusqu'aux massifs ampliata. Passe du bleu clair au vert et au bronzé.

5. Aurigena lugubris Oliv., Ent., t. 2 (1790), gen. 32, p. 60, pl. 10 , f. 106 . - A peu près le même habitat que celui de Capnodis tenebrionis L. - Pris en quantité dans la Luristan (Perse).

6. Aurigena cuprata Klug, Symb. Phys., t. 1 (1829), pl. 2, f. 5. - Perse: Luristan. Signalé dans le Caucase et en Syrie.

7. Capnodis tenebrionis Linné, Syst. Nat. Ed. X, t. 1 (1758), p. 661. - Perse: Luristan. L'espèce est commune dans tout le bassin de la mer Méditerranée.

8. Capnodis anthracina Fisch., Bull. Soc. Nat. Mosc. (1830), p. 184. - Perse: Luristan, quelques exemplaires.

9. Sphenoptera semistriata Palis., Ins. d'afr. (1807), p. 43, pl. 11, f. 1. - Perse : Luristan.

10. Sphenoptera Mniszechi Mars., L'Abeille, t. 2 (1865), p. 332. Perse: Luristan.

11. Sphenoptera elamita Mars., 1. c., p. 338. - Perse: Luristan, Prise en grande quantité.

12. Sphenoptera coracina Stev., Nouv. Mém. Mosc., t. 2 (1830), p. 2, pl. 3, f. 5. - Perse: Luristan, 4 exemplaires.

13. Sphenopterafossa n. sp. - Long. 13, larg. 5 mm.

Bronzé obscur, presque noir en dessus; dessous noir. Tête finement pointillée, la ponctuation plus dense vers l'épistome; épistome faiblement échancré en arc; front légèrement déprimé avec deux cicatrices subgemellées surmontées d'un court et fin sillon; vertex avec un très court sillon contre la marge antérieure du pronotum; Antennes courtes, à $3^{\mathrm{e}}$ article deux fois plus long que le $2^{\mathrm{e}}$. Pronotum inégal, transversal, bisinué en avant, droit 
sur les côtés avec les angles antérieurs arrondis, fortement bisinué à la base avec le lobe médian largement tronqué; le sillon médian net, et linéaire au dessus de l'écusson s'élargit ensuite et devient plus vague et moins profond; des deux côtés du sillon une fossette discale arrondie et une profonde impression, sous la fossette et prolongée en arrière vers l'écusson, suivant un sillon arqué. Des deux côtés, le long de la marge latérale, deux autres impressions superposées; marge antérieure fine, non prolongée vers le milieu; carène marginale interrompue au tiers antérieur et presque droite; la surface assez lisse, à fine ponctuation assez espacée. Écusson plus large que haut, transversalement et nettement sillonné. Elytres légèrement gondolés sur les côtés, c'est à dire déprimés sous le calus huméral et au tiers postérieur, obliquement et faiblement atténués en ligne droite de la base au tiers postérieur; le sommet largement et peu obliquement tronqué avec ses côtés externes arrondis; strie marginale dépassant à peine le milieu, le rebord marginal visible en dessus et saillant à la hauteur des hanches postérieures; largement déprimés à la région scutellaire, étroitement le long de la suture, au sommet; la surface couverte de séries longitudionales de points formant de petits traits espacés, plus nets sur la moitié antérieure que sur la postérieure, leurs intervalles avec une ou deux séries de points très fins. Strie marginale du prosternum non continuée en arrière et formée de points irréguliers; pas de sillon abdominal ni de plaques lisses sur les côtés, ceux ci granuleux et couverts d'une pubescence jaune; le milieu se l'abdomen à peine ponctué, sauf sur le dernier segment, où la ponctuation est assez dense, nette et régulièrement espacée. Pattes grossièrement ponctuées.

Faciès rappellant celui des S. elamita Mars. et coracina Stev., un peu moins robuste, moins rugueux en dessus, la structure du pronotum entièrement différente.

Perse : Luristan.

14. Sphenoptera glabrata Ménétr., Cat. Rais. (1832), p. 151. Perse : Luristan.

15. Sphenoptera foveola Gebler, Humm. Ess., t. 4 (1825), p. 46. Perse: Luristan.

16. Sphenoptera morio Jakowl., Hor. Soc. Ent. Ross., t. 20 (1886), p. 84. - Perse: Luristan, 4 exemplaires.

17. Sphenoptera aethiops Jakowl., 1. c., t. 25 (1890), p. 129. Perse: Luristan, 4 exemplaires.

18. Sphenopteraluristana nov. sp. - Long. 18, larg. $6 \mathrm{~mm}$.

Oblong ovale, allongé, arrondi en avant et atténué en arrière, bronzé obscur mais brillant en dessus avec l'épistome et le sommet 
des élytres légèrement teintés de bleu d'acier; dessous noir bleuâtre brillant avec les côtés d'un cuivreux pourpré violacé sous une vestiture brun jaunâtre. Tête unie et plane, à ponctuation excessivement fine, irrégulièrement espacée et peu dense ; épistome subanguleusement échancré; $3^{\mathrm{e}}$ article des antennes deux fois aussi long que le $2{ }^{\mathrm{e}}$. Pronotum transversal à côtés droits et parallèles, arrondis en avant; la marge antérieure bisinuée et a strie marginale entière; la carène latérale étroite, subsinueuse et atteignant presque l'angle antérieur; la base bisinuée avec le lobe médian tronqué; la surface unie, sans sillons ni dépressions autres qu'une faible fossette antéscutellaire et couverte d'un pointillé excessivement fin alternant avec des points plus forts, assez également distants et plus épais sur les côtés. Ecusson très large, subpentagonal, un peu concave. Elytres allongés, déprimés à la région suturale, largement et séparément arrondis au sommet; étroitement rebordés sur les côtés de la base au sommet; la surface couverte de séries longitudinales de traits allongés, dont les intervales sont bisérialement mais inégalement pointillés, ces points excessivement fins. Prosternum sans strie marginale, avec trois ou quatre gros points latéraux irréguliers; milieu du premier segment abdominal sillonné; ponctuation abdominale rare, inégale et irrégulière, sauf sur le dernier segment où elle est plus dense; pas de plaques lisses sur les côtés. Pattes grossièrement ponctuées.

Faciès de $C$. aethiops, mais plus robuste, plus acuminé en arrière; très différent de lui par la structure élytrale.

Perse : Luristan.

19. Sphenoptera cribrata Fald., Nouv. Mém. Mosc., t. 4 (1835), p. 152 , pl. 5, f. 4. - Perse : Luristan, un seul exemplaire.

20. Sphenoptera antiqua Illig., Mag. Ins., t. 2 (1803), p. $247=$ Babel Mars., L'Abeille, t. 2 (1865), p. 340. - Asie mineure: Eski-Chehir, en très grande quantité.

\section{Sphenoptera Rangnowi nov. sp.}

Long. 11-17, larg. 3,5-6,5 mm.

Ovalaire, allongé, atténué en arrière, d'un noir intense en dessus; dessous bleu foncé brillant. Tête finement pointillée; front garni de deux reliefs obliques à peine sensibles; vertex finement sillonné, le sillon prolongé en avant sur le front; $3^{\mathrm{e}}$ article des antennes deux fois plus long que le $2^{\mathrm{e}}$. Pronotum plus large que haut, atténué en avant, arrondi sur les côtés antérieurs, droit ensuite à partir du milieu; la marge antérieure entièrement striée et finement rebordée; la carène marginale fine, un peu sinu- 
euse et interrompue au tiers antérieur; la base bisinuée avec le lobe médian tronqué; le disque avec une fossette antéscutellaire prolongée en avant suivant un vague sillon, assez large, interrompu un peu au delà du milieu; la surface couverte d'une ponctuation très fine et très espacée sur le disque et sur les côtés, plus épaisse des deux côtés de la région médiane. Ecusson large, tronqué en avant, arrondi sur les côtés, bisinué et acuminé en arrière. Elytres déprimés à la région scutellaire, régulièrement atténués en arc de la base au sommet, celui-ci arrondi sur les côtés et en angle droit à la suture avec un petit vide anguleux sutural; strie marginale interrompue avant le milieu; la surface à peine ponctuée, la ponctuation tellement vague et confuse qu'on en distingue à peine l'allure longitudinale, le disque couvert ça et là de petits traits superficiels et formant de fines marbrures. Strie marginale du prosternum nulle, remplacée par quelques gros points irréguliers; milieu du premier segment abdominal sans sillon; côtés de l'abdomen et des hanches ni pointillés ni villeux; ponctuation abdominale fine et rare. Pattes grossièrement ponctuées.

Voisin du S. lia Jakowl., mais différent de celui ci par la forme du pronotum dont les angles postérieurs ne débordent pas sur les élytres et par une série de détails secondaires. D'un noir plus intense en dessus, à peine bleuâtre; le dessin élytral tout autre.

Perse: Luristan; vingt quatre exemplaires.

22. Sphenoptera subtilis Jakowl., Hor. Soc. Ent. Ross., t. 32 (1894), p. 552. - Perse: Luristan; Nombreux exemplaires.

23. Sphenoptera cuprifrons Fald., Nouv. Mém. Mosc., t. 4 (1835), p. 153 , pl. 5, f. 5. - Luristan (Perse); en grande quantité. Le nom choisi pour cette espèce, par F a l d e rmann, n'est pas tout à fait conforme à la réalité. Bien peu d'exemplaires ont le front cuivreux, la coloration de cette partie de la tête étant très variable.

24. Sphenoptera Bodemeyeri Jakowl., Hor. Soc. Ent. Ross., t. 34 (1900), p. 501. - Asie mineure: Eski-Chehir.

25. Sphenoptera coemulea Jakowl., Hor. Soc. Ent. Ross., t. 32 (1898), p. 559. - Perse: Juristan, une douzaine d'exemplaires.

26. Sphenoptera quadrifoveolata Mars., L'Abeille, t. 2 (1865), p. 356. - Perse: Luristan, 2 exemplaires. 
27. Sphenoptera obruta nov. sp. - Long. 14, larg. $5,5 \mathrm{~mm}$. Ovalaire, écourté, assez convexe, bronzé cuivreux obscur en dessus; dessous bronzé noirâtre. Tête peu convexe, couverte de points inégalement espacés entre de vagues reliefs peu saillants; épistome étroitement et profondément échancré en arc ; $3^{\mathrm{e}}$ article des antennes une fois et demi aussi long que le $2^{\mathrm{e}}$. Pronotum convexe, plus large que long, bisinué en avant avec une strie marginale entière, arqué sur les côtés avec la carène marginale un peu arquée et atteignant presque les angles antérieurs; la base bisinuée avec le lobe médian tronqué; la surface également convexe, à peine déprimée au milieu, couverte d'une fine ponctuation très espacée sur le disque et s'épaississant sur les côtés. Ecusson large, subcordiforme, plan. Elytres plans sur le disque, déclives sur les côtés, à peine déprimés sur la région scutellaire, graduellement atténués de la base au sommet avec le rebord marginal visible en dessus jusque vers le tiers postérieur; pas de strie marginale, mais un fin rebord lisse; le sommet largement arrondi, subtronqué obliquement; la surface couverte de séries longitudinales de traits fins, les intervalles assez grossièrement pointillés en avant, plus finement en arrière. Prosternum sans strie marginale; celle ci remplacée par une série de points; milieu du premier segment abdominal sillonné; côtés des segments abdominaux sans plaques lisses, mais couverts d'une pubescence jaunâtre; ponctuation abdominale superficielle, subocellée et subaciculée. Pattes grossièrement ponctuées; tibias antérieurs à peine arqués, les médians droits.

Faciès de $S$. abbreviata Jakowl., mais la ponctuation élytrale tout autre, la coloration du dessous différente.

Syrie.

28. Sphenoptera cyaneoniger nov. sp.

Long. 10,5, larg. $4 \mathrm{~mm}$.

Ecourté, ovalaire, assez convexe en dessus; tête bleue avec les mandibules et l'épistome cuivreux pourpré; pronotum noir à léger reflet pourpré; élytres noir bleuâtre. Dessous noir brillant avec les côtés vaguement cuivreux pourpré. Tête assez fortement ponctuée; épistome étroitement échancré en arc; front déprimé ; vertex faiblement sillonné; $3^{\mathrm{e}}$ article des antennes un peu plus long que le $2^{\mathrm{e}}$. Pronotum plus large que haut, à peine plus étroit en avant qu'en arrière, l'angle antérieur brusquement déclive; la marge antérieure bisinuée et finement rebordée avec une strie marginale entière; le disque vaguement déprimé; la surface couverte d'une ponctuation assez dense, plus fine et plus espacée sur le disque; la base bisinuée avec le lobe médian tronqué. 
Ecusson large, en losange, anguleux sur les côtés. Elytres écourtés, largement arrondis en arrière, régulièrement atténués en arc de la base du sommet; la marge latérale finement rebordée jusqu'au quart postérieur; la région scutellaire et la terminale faiblement déprimées le long de la suture; la surface couverte de séries longitudinales de points assez forts et assez rapprochés mais inégaux, les intervalles à points plus faibles, mais assez accusés. Prosternum sans strie marginale, celle-ci remplacée par quelques points irréguliers; milieu du premier segment abdominal plan; abdomen assez régulièrement et visiblement ponctué. Pattes grossièrement ponctuées; tibias antérieurs et les médians très arqués $\sigma^{7}$.

Faciès de $S$. coerulea Jakowl., mais plus rugueux en dessus, moins convexe et un peu plus trapu.

Asie mineure: Karabunar.

29. Sphenoptera abbreviata Jakowl., Hor. Soc. Ent. Ross., t. 34 (1899), p. 104. - Perse: Luristan, en assez grande quantité.

30. Sphenoptera interrupta nov. sp. - Long. 20, larg. $7 \mathrm{~mm}$.

Robuste, ovalaire, assez convexe, entièrement d'un bronzé cuivreux clair et brillant; antennes et tarses obscurs. Tête assez grossièrement ponctuée, les intervalles formant des reliefs vermiculés et longitudinaux ; front déprimé ; vertex sillonné ; épistome allongé et faiblement échancré en arc; $3^{\mathrm{e}}$ article des antennes deux fois aussi long que le $2^{\mathrm{e}}$ et plus long que le $4^{\mathrm{e}}$. Pronotum grand, convexe en avant, largement déprimé en arrière au milieu; la marge antérieure bisinuée et finement rebordée; les côtés largement arrondis en avant, sinueux en arrière avec l'angle postérieur légèrement saillant en dehors; la carène latérale visible en partie en dessus, subsinueuse et atteignant presque l'angle antérieur; la base bisinuée avec le lobe médian largement arqué; le surface couverte de points assez faibles et très espacés sur le disque, très rapprochés, très épais, subconfluents sur les côtés. Ecusson deux fois et demie plus large que haut, en trapèze élargi en arrière. Elytres graduellement atténués en arc de la base au sommet, celui ci arrondi latéralement et obliquement tronqué ensuite avec une très courte dent suturale; les côtés finement rebordés jusque vers le sommet; le disque vaguement déprimé; la surface couverte de stries linéaires fines, nettes, très irrégulièrement interrompues; les interstries plans, couverts de points fins, alternativement disposés en double série. Prosternum sans strie marginale; premier segment abdominal sans sillon médian; ab- 
domen irrégulièrement ponctué. Pattes grossièrement ponctuées ; tibias antérieurs à peine arqués, les médians droits ㅇ.

Turkestan.

31. Sphenoptera uncta nov. sp. - Long. 19, larg. $7 \mathrm{~mm}$.

Robuste, élargi, peu convexe, d'un bronzé clair et brillant en dessus; dessous bronzé violacé brillant. Tête déprimée; vertex finement sillonné ; épistome échancré en arc, à ponctuation épaisse ; $3^{\mathrm{e}}$ article des antennes une fois et demie aussi long que le $2^{\mathrm{e}}$ et que le $4^{\mathrm{e}}$. Pronotum large, déprimé au milieu et sur les côtés; la marge antérieure striée sur les côtés, à peine rébordée au milieu; les côtés arrondis en avant et droits en arrière avec la carène marginale arquée et interrompue en avant; le milieu du disque largement déprimé au dessus de l'écusson, la dépression grossièrement et densément ponctuée; le parties bordant la dépression presque lisses, avec quelques points épars; les dépressions latérales allongées, limitées par un bourrelet latéral, couvertes, ainsi que ces bourrelets, d'une épaisse ponctuation à points superficiels; la base bisinuée avec un large lobe médian arqué. Ecusson large, transversalement convexe, trapézoïdal. Elytres semblables, pour la forme, la structure et la sculpture, à ceux de S. Gebleri Cast. et Gory. Prosternum sans strie marginale; milieu du premier segment abdominal non sillonné; abdomen presque lisse, très finement ponctué. Pattes grossièrement ponctuées; tibias antérieur et médian arqués.

Ressemble beaucoup au $S$. Gebleri Cast. et Gory, mais plus robuste, plus large, d'un bronzé moins pourpré clair et brillant en dessous, la dépression du disque du pronotum plus arrondie, non allongée ni sillonnée dans le fond.

Perse: Luristan; un seul exemplaire.

32. Sphenoptera Gebleri Cast. et Gory, Monogr., t. 2 (1839), p. 12, pl. 3, f. 14. - Perse: Luristan, 4 exemplaires.

33. Sphenoptera profus a nov. sp. - Long. 13, larg. $4 \mathrm{~mm}$.

Allongé, peu convexe, arrondi en avant, atténué en ligne droite en arrière, d'un bronzé obscur légèrement pourpré en dessus ; dessous noir brillant, légèrement bleuté, avec les côtés du sternum et des segments abdominaux cuivreux pourpré brillant. Tête ponctuée; front séparé de l'épistome par un relief à contours bisinués qui font paraître le second sur un plan inférieur au premier; épistome faiblement échancré en arc. Pronotum assez convexe, plus large que haut, bisinué en avant avec une strie marginale distincte sur les côtés; les côtés arrondis en avant et presque 
parallèles, faiblement atténués vers la base avec la carène marginale épaisse, sinueuse et visible en dessus; la base fortement bisinuée avec le lobe médian large, obliquement cintré de part et d'autre; le disque avec trois sillons, le médian étroit, profond et allant de la base au tiers antérieur, les latéraux arqués, parallèles au bord extérieur et moins avancés vers le sommet que le médian, la carène marginale elle-même bordée intérieurement d'un sillon; la surface à peine ponctuée sur le disque, plus sensiblement entre les sillons latéraux et le bord. Ecusson cordiforme, élargi, tronqué à la base. Elytres finement rebordés jusque vers le sommet, couverts de séries longitudinales de points bien marqués, les interstries sans points distincts, ceux de la base relevés en bourrelets assez saillants; le $3^{\text {e }}$ et l'avant dernier vaguement relevés en côtes; le sommet largement arrondi, subtronqué. Strie marginale du prosternum remplacée par une série de points; milieu du premier segment abdominal non sillonné; ponctuation abdominale à peine distincte. Pattes grossièrement ponctuées; tibias antérieurs et médians arqués, $\sigma^{7}$.

$\mathrm{Du}$ groupe des espèces rappelant le $S$. lapidaria Brullé, mais entièrement distinct de cette espèce.

Perse: Luristan; un seul exemplaire.

34. Sphenoptera prava nov. sp. - Long. 14, larg. $5 \mathrm{~mm}$.

Ovalaire, écourté, convexe, atténué en arrière, bronzé brillant obscur en dessus; dessous noir bleuâtre, avec les côtés pubescents de jaune terne. Tête rugueuse, grossièrement ponctuée, transversalement déprimée entre le front et l'épistome; le front et le vertex sillonnés; l'épistome étroitement échancré en arc; $3^{\mathrm{e}}$ article des antennes une fois et demie aussi long que le $2^{\mathrm{e}}$. Pronotum plus large que haut, bisinué en avant et très finement rebordé avec la strie marginale à peine distincte; les côtés arqués en avant et un peu sinueux en arrière avec la carène marginale subsinueuse et atteignant presque le sommet; la base faiblement sinueuse; le disque et les côtés largement creusés en sillon; le médian inégalement ponctué, les latéraux rugueux, .grossièrement ponctués entre d'épaisses vermiculations. Ecusson subcordiforme, déprimé. Elytres écourtés, convexes, très rugueux, graduellement atténués en arc de la base au sommet, celui-ci obliquement tronqué de part et d'autre; la région scutellaire déprimée, la suture élevée au sommet; la surface à rides vermiculées transversales et épaisses, laissant à peine distinguer l'allure longitudinale de traits minces et linéaires. Strie marginale du prosternum formée de gros points confluents en arrière, mais sans contourner le sommet; milieu du premier segment abdominal vaguement et étroitement sillonné; 
ponctuation abdominale assez nette, mais très espacée. Pattes grossièrement ponctuées; tibias antérieurs et médians arqués, ơ

Asie mineure: Eski-Chehir.

35. Sphenoptera torrida Jakowl., Hor. Soc. Ent. Ross., t. 32 (1898), p. 553. - Perse: Luristan, 4 exemplaires.

36. Sphenoptera ambigua Klug, Symb. Phys., t. 1 (1829), p. 28, pl. 3, f. 2. - Perse: Luristan, 2 exemplaires.

37. Sphenoptera quadrata nov. sp. - Long. 9, larg. 2,5 mm.

Oblong allongé, peu convexe, atténué en arrière, d'un bronzé clair et brillant en dessus; dessous bronzé obscur, presque noir, les côtés pubescents de jaune cendré. Tête large, peu convexe, finement ponctuée, plus grossièrement en avant; épistome très court, subanguleusement échancré; front impressionné entre les yeux; vertex non sillonné. Pronotum grand, subrectangulaire, presqu'aussi large que haut, élargi sur les côtés en avant, rétréci et sinueux en arrière; la marge antérieure faiblement bisinuée, très étroitement rebordée et striée sur les côtés seulement; carène marginale subsinueuse, interrompue en avant, visible en dessus vers la base; le milieu du disque sans sillon, les côtés du disque transversalement déprimés, formant, avec la sinuosité des côtés, une sorte d'étranglement peu prononcé; la surface couverte de points fins, un peu plus épais sur les côtés; la base bisinuée avec le lobe médian tronqué. Ecusson large, subcordiforme, tronqué en avant, bisinué et acuminé en arrière. Elytres couverts de séries longitudinales de points alternant avec de vagues côtes élevées; la région scutellaire et la suturale extrème faiblement déprimées; le sommet obliquement tronqué et inerme. Prosternum et premier segment abdominal vaguement sillonnés au milieu, le premier sans strie marginale distincte. Abdomen finement, pattes grossièrement ponctuées; tibias antérieurs presque droits, les médians droits, $q$.

Asie mineure: Eski-Chehir.

38. Sphenoptera delicata nov. sp. - Long. 8, larg. $3,5 \mathrm{~mm}$.

Oblong ovale, écourté, assez convexe, bronzé clair et brillant en dessus; dessous d'un bronzé plus terne et plus obscur, légèrement cuivreux pourpré sur les côtés; tarses et antennes noirs. Tête densément ponctuée, avec deux faibles reliefs obliques et lisses au milieu du front; épistome très court, étroitement échancré en arc; vertex non silonné; $3^{\text {e }}$ article des antennes plus long que le $2^{\mathrm{e}}$ et plus court que le $4^{\circ}$. Pronotum grand, uni, convexe, sans traces d'impressions ou de sillons-quelconques; la 
marge antérieure sans rebord ni stries, distincts; les côtés faiblement arqués en avant et à peine sinueux en arrière; avec la carène marginale 'subsinueuse, à peine visible en dessus et presque entière; la base bisinuée avec le lobe médian tronqué; la surface très finement ponctuée sur le disque, plus grossièrement sur les côtés. Ecusson large, subcordiforme, tronqué à la base. Elytres écourtés, assez rugueux, plissés à la base, conjointement arrondis au sommet et inermes; la suture éleéve sur toute la moitié postérieure; la surface vaguement plissée dans le sens de sa longueur, les points indistincts, remplacés par des rugosités subtransversales. Prosternum large et plan, avec une strie marginale sur les côtés seulement; milieu du premier segment abdominal non sillonné; hanches postérieures échancrées sur le bord inférieur, plus près de la marge latérale que du milieu; abdomen sensiblement ponctué. Pattes grossièrement ponctuées; tous les tibias droits, +.

Asie mineure: Eski-Chehir.

39. Sphenoptera cunea Mars., L'Abeille, t. 2 (1865), p. 346. Perse: Luristan, 8 exemplaires.

40. Sphenoptera subchalybea nov. sp.

Long. 14, larg. 5,5 mm.

Ovalaire, peu convexe, assez robuste, d'un gris de plomb bleuâtre en dessus; dessous noir brillant. Tête assez densément ponctuée avec deux vagues reliefs lisses surmontés chacun d'une fossette peu profonde; vertex finement sillonné; épistome court, faiblement échancré en arc. Pronotum plus large que haut, arrondi sur les côtés en avant, droit en arrière; la marge antérieure bisinuée, rebordée et striée sur les côtés seulement; la carène marginale presque nulle, sensible seulement à la base; la base bisinuée avec le lobe médian tronqué; le disque nettement sillonné au milieu sur toute sa longueur, le sillon terminé en arrière par une fossette préscutellaire; deux fossettes de chaque côté, du sillon, une dans chacun des angles postériers et une, de chaque côté, au dessus des précédentes vers l'angle antérieur : toutes ces fossettes peu accentuées; la surface couverte d'une ponctuation assez épaisse et assez également espacée, plus épaisse et subsquameuse sur les côtés. Ecusson cordiforme, très large. Elytres droits sur les côtés jusqu'au tiers postérieur, ensuite atténués en arc jusqu'au sommet; celui-ci obliquement tronqué et inerme; les côtés rebordés avec une strie marginale distincte jusque vers le tiers postérieur, le rebord marginal visible en dessus sur toute sa moitié antérieure; la surface 
couverte de séries gémellées de points dont les intervalles sont relevés en côtes. Prosternum large, sans strie marginale, mais avec quelques points inégaux sur les côtés; milieu du premier segment abdominal sans sillon; abdomen finement ponctué. Pattes grossièrement ponctuées; tibias antérieurs faiblement arqués, les médians droits, or.

Turkestan; un seul exemplaire.

41. Sphenoptera trisulcata Reiche, ann. Fr. (1856), p. 413. -Perse: Luristan.

42. Sphenoptera os tentator nov. sp. - Long. 14, larg. 5 mm.

Ovalaire, très atténué en arrière, peu convexe, mais déclive en arrière, d'un bronzé cuivreux clair et brillant en dessous avec l'épistome et les bords latéraux du pronotum cuivreux pourpré; dessous pourpré brillant avec les trois derniers segments abdominaux bleu d'acier; antennes et tarses obscurs. Tête assez fortement et inégalement ponctuée; front impressiorné en avant de deux vagues reliefs lisses; vertex sillonné; épistome étroitement et peu profondement échancré en arc; $2^{\mathrm{e}}$ article des antennes presqu'aussi long que le $3^{\mathrm{e}}$. Pronotum grand, plus large que haut, bisinué en avant avec un rebord et une strie marginale distincts sur les côtés, nuls au milieu; les côtés très arqués en avant et rentrants en arrière, leur plus grande largeur avant le milieu, la carène marginale fine, sinueuse et limitée par une fine strie prémarginale; la base bisinuée avec un large lobe médian arqué; le disque avec une large dépression médiane, ovalaire et amincie en avant et deux larges sillons latéraux, ceux-ci limités extérieurement par un large bourrelet latéral; la surface à peine ponctuée, presque lisse sur les reliefs limitant la dépression médiane, celleci à gros points inégalement espacés; les sillons latéraux plus grossièrement et plus densément ponctués que les bourrelets latéraux qui les limitent. Ecusson en cœur élargi. Elytres allongés, régulièrement atténués en arc de la base au sommet; celui-ci obliquement tronqué de part et d'autre et inerme; la surface couverte de séries géminées de gros points alternant avec des rides transversales, ces séries de points alternant avec la suture, deux côtes discales entières, une côte prémarginale plus courte et une côte marginale; la suture élevée sur toute son étendue; la région scutellaire déprimée et sillonnée. Strie marginale du prosternum figurée par quelques points; abdomen non sillonné au milieu du premier segment, à ponctuation rare et très fine. Pattes grossièrement ponctuées; tibias antérieurs et médians arqués. - Syrie; un seul exemplaire. 
43. Sphenoptera lapidaria Brullé, Exp. Morée (1832), p. 134, pl. 35, f. 1. - Asie mineure: Eski-Chehir, 4 exemplaires.

44. Sphenoptera Curceli Cast. \& Gory, Monogr. t. 2 (1839), p. 21, pl. 5, f. 29 . - Asie mineure: Eski-Chehir, 4 exemplaires.

45. Sphenoptera subsulc a ta nov. sp. - Long. 8, larg. 2,5 mm.

Ovale oblong, atténué en arrière, bronzé brillant, presque noir en dessus; dessous d'un bronzé plus clair, moins brillant. Tête inégale, assez grossièrement ponctuée, avec deux reliefs frontaux vagues et irréguliers; vertex finement sillonné; épistome court, faiblement échancré en arc. Pronotum assez convexe, un peu plus large que haut, bisinué en avant sans rebord ni strie marginale distincts; la marge latérale avec une carène sinueuse n'atteignant pas le sommet; les côtés arrondis en avant et droits en arrière; la base bisinuée avec le lobe médian échancré en arc; le disque avec trois sillons longitudinaux très vagues, le médian assez large, sensible seulement en avant et en arrière; sans fossette préscutellaire, les latéraux formés par des amas de points rapprochés; la surface finement granuleuse avec quelques points espacés. Ecusson subcordiforme, la base arquée et insérée dans l'échancrure du lobe médian de la base du pronotum. Elytres rebordés jusque près du sommet, le rebord visible en dessus sur toute son étendue; presque droits sur les côtés jusqu'au tiers postérieur, atténués ensuite, séparément arrondis au sommet avec une très petite dent suturale; la surface subcostiforme entre de très vagues séries de points et de costules transversales à peine visibles; la région scutellaire non déprimée; la région suturale creusée en arrière avec la suture relevée du tiers antérieur au sommet. Strie marginale du prosternum interrompue au sommet; milieu du premier segment abdominal vaguement sillonné; abdomen assez densément ponctué. Pattes grossièrement ponctuées; tibias antérieurs et médians droits.

Perse: Luristan.

46. Sphenoptera lineola Herbst, Col., t. 9 (1801), p. $284=$ ge minata Illig., Mag. Ins., t. 4 (1803), p. 244. France méridionale, 2 exemplaires.

47. Sphenoptera Koenigi Jakowl., Hor. Soc. Ent. Ross., t. 25 (1890), p. 133. - Perse: Luristan, très nombreux exemplaires.

48. Sphenoptera acuminata Jakowl., 1. c., t. 32 (1898), p. 331. Perse: Luristan, une douzaine d'exemplaires.

49. Sphenoptera excavata Fald., Faun. Transc., t. 1 (1835), p. 154, pl. 5, f. 7. - Perse: Luristan, 2 exemplaires. 
50. Sphenoptera clarescens nov. sp. - Long. 9, larg. $3 \mathrm{~mm}$.

Oblong ovale, allongé, peu convexe, atténué en arrière d'un bronzé clair en dessus; dessous bronzé obscur; tarses et antennes noirs. Tête assez large, finement pointillée en arrière, plus grossièrement en avant; le front avec deux faibles reliefs obliques; le vertex finement sillonné; l'epistome étroitement et faiblement échancré. Pronotum un peu plus large que haut, faiblement arqué sur les côtés en avant, droit ensuite jusque la base; la marge antérieure rebordée sur les côtés, sans strie distincte au milieu; la carène marginale subsinueuse, atteigant presque le sommet et visible en dessus sur presque toute son étendue; la base bisinuée avec le lobe médian faiblement échancré ; le disque sans sillon longitudinal, mais avec une fossette préscutellaire et, de chaque côté, une vague dépression transversale; la surface couverte de points fins, également espacés et un peu plus denses et plus épais sur les côtés que sur le disque. Ecusson large, subcordiforme, tronqué en avant, sinué et acuminé en arrière. Elytres graduellement atténués en arc de la base au sommet, carénés et striés le long du bord externe sur toute leur étendue, le rebord entièrement visible en dessus; le sommet sinueux de part et d'autre avec une très courte dent suturale et une dent obtuse, plus courte encore, à la limite externe du sinus terminal; la surface finement granuleuse avec des séries de points assez rapprochés et assez épais, sans côtes distinctes sauf vagues costules subterminales limitant deux vagues sillons suturaux; la suture élevée dans sa moitié postérieure; la région scutellaire plane. Prosternum muni d'une strie marginale entière, contournant le sommet; milieu du premier segment abdominal vaguement sillonné; abdomen assez grossièrement ponctué. Pattes plus rugueuses; tibias antérieurs et médians droits; les postérieurs légèrement arqués en dehors.

Perse: Luristan; un seul exemplaire.

51. Sphenoptera saxosa nov. sp. - Long. 9, larg. 2,5 mm,

Ovale oblong, peu convexe, atténué en arrière, d'un noir intense et brillant en dessus avec les élytres très rugueux; dessous bleu foncé très brillant. Tête assez large, un peu convexe, finement ponctuée en arrière, un peu plus grossièrement en avant, avec deux reliefs frontaux; vertex sillonné; épistome étroitement et assez profondément échancré en arc; $3^{\mathrm{e}}$ article des antennes une fois et demie aussi long que le $2^{\ominus}$. Pronotum subrectangulaire, légèrement élargi en avant; la marge antérieure bisinuée, entièrement rebordée avec une très fine strie marginale; les côtés 
arqués en avant, obliquement atténués en ligne droite en arrière, avec une carène marginale sinueuse, atteignant presque le sommet et invisible en dessus; la base bisinuée avec un lobe médian tronqué; le disqué sillonné au milieu et sur les côtés, le sillon médian entier mais peu profond, terminé en arrière par une profonde fossette préscutellaire; les lateraux plus nets, plus larges, grossièrement ponctués et approfondis dans leur milieu; la surface couverte de points fins et espacés sur le disque, très épais et profonds sur les côtés. Ecusson transversal elliptique, convexe, acuminé sur les côtés et en arrière. Elytres graduellement atténués de la base au sommet, celui-ci séparément arrondi et inerme; la surface couverte de séries de points linéaires interrompus par d'épāis reliefs vermiculés. Prosternum à strie marginale entière et contournant le sommet; milieu du premier segment abdominal à peine sillonné; abdomen finement ponctué. Pattes grossièrement ponctuées; tibias antérieurs et médians arqués, $\sigma^{7}$.

Perse: Luristan; un seul exemplaire.

52. Sphenoptera Tappesi Mars., L'Abeille, t. 2 (1865), p. 387. Asie mineure: Eski-Chehir; un seul exemplaire. Le type provient de la Turquie.

\section{Zwei neue neotropische Megacephalae. (Col.) Von Walther Horn, Berlin.}

Megacephala (Phaeoxantha) bucephala n. sp.

$P h$. Klugi Chaud. affinis, differt $q \sigma^{7}$ capite prothoraceque multo latioribus, hoc postice evidenter magis constricto; elytris multo brevioribus, antice aeque latis atque illa specie ante apicem; elytrorum margine apicali non dentato, impressione discoidali ante apicem leviore, superficie paullo magis nitente perparumque minus confluenter sculpta. $\sigma^{7}$ dente (praeter apicem) penultimo mandibulae dextrae lato brevi obtuso; elytrorum dimidia parte postica perparum dilatata, margine libero ad angulum apicalem externum rotundato-declivi vixque tumido, sulculo levi juxtamarginali hoc loco nec dislocato nec interrupto (in Ph. Klugi Chaud. hac in parte margine tumido perparumque declivi et sulculo juxtamarginali intus [discum] versus dislocato paullulumque obliterato). q elytris in medio perparum dilatatis, pone medium angustatis. Long. 21-25 mm (sine labro): maxima prothoracis latitudine 7 (q) ad $9\left(\sigma^{\top}\right) \mathrm{mm}$, maxima elytrorum latitudine $8^{1 / 2}$ (q) ad $10^{1 / 2}\left(\sigma^{7}\right) \mathrm{mm}$. 


\section{$2 \mathrm{BHL}$ Biodiversity Heritage Library}

Kerremans, Ch. 1909. "Buprestides recueillis par M. E. von Bodemeyer en Perse et en Asie mineure." Deutsche entomologische Zeitschrift 1909(2), 270-284. https://doi.org/10.1002/mmnd.48019090210.

View This Item Online: https://www.biodiversitylibrary.org/item/103377

DOI: https://doi.org/10.1002/mmnd.48019090210

Permalink: https://www.biodiversitylibrary.org/partpdf/235945

\section{Holding Institution}

Harvard University, Museum of Comparative Zoology, Ernst Mayr Library

\section{Sponsored by}

Biodiversity Heritage Library

\section{Copyright \& Reuse}

Copyright Status: Public domain. The BHL considers that this work is no longer under copyright protection.

This document was created from content at the Biodiversity Heritage Library, the world's largest open access digital library for biodiversity literature and archives. Visit BHL at https://www.biodiversitylibrary.org. 